\title{
COVID-19 Quarantine Monitoring Based on Geofencing Technique
}

\author{
Nawar Alqrnawi*, Indrit Myderrizi** \\ * Institute of Graduate Studies, Electrical and Electronics Engineering Program, Istanbul Gelisim University, Istanbul, Turkey \\ ** Department of Electric \& Electronics Engineering, Faculty of Engineering and Architecture, İstanbul Gelisim University, \\ Istanbul, Turkey \\ (alqrnawinawar@gmail.com,imyderrizi@gelisim.edu.tr) \\ †Nawar Alqrnawi; Indrit Myderrizi, Tel: +90 21242270 00, \\ alqrnawinawar@gmail.com
}

Received: 16.06.2021 Accepted: 18.06.2021

\begin{abstract}
Smartphone and satellite network coverage contributes to a number of useful applications that increase the comfort of everyday people's lives. Using the Global Positioning System (GPS) provided by the satellite, people can be tracked in real time at a specific location. When it comes to infectious diseases such as COVID-19, the most important measure to reduce its spread is to contain it. Quarantine is usually a solution and any tracking system can be employed to specify the location of the infected people. However, as quarantined areas are scattered around the world, it is difficult to accurately trace the boundaries within accommodation. A geo-fenced GPS system, which has adopted an efficient border protection system, is proposed for quarantine and monitoring purposes of COVID-19 carriers. The system is introduced as an application to implement a home/local quarantine policy using bracelets that fit the boundaries of smartphones. From the experimental results obtained, it is observed that the proposed system achieves an effective and high accuracy performance in various location conditions.
\end{abstract}

Keywords- Quarantine; tracking system; GPS; GSM.

\section{Introduction}

The fact that Coronavirus Disease 2019 (COVID-19) is highly contagious has been an epidemic that has had a profound impact on people's lives and the economies of countries [1]. Therefore, prevention of infection by COVID-19 is the first step in effectively curbing its spread. People who have traveled from risky areas and had physical contact with infected cases are expected to be isolated and placed in quarantine areas for a period of 10-14 days. In many countries, mandatory quarantine orders are issued for those coming from abroad, whether at home or in care centers. Any breach of home quarantine (i.e. during the quarantine period when the infected person leaves the home) can pose a great risk and expense to public health.
Various technologies have been developed to locate any object. There are differences between these technologies, including cost, precision, functionality, scope, and coverage required to operate them, but choosing the right technique depends on the application or task required. Tracking infected individuals effectively and continuously is a challenge. One of the successful solutions has been to provide periodic satellite remote control reports on the location of infected people. Such reports can be provided daily and/or instantly by sending them to remote control devices based on satellite systems such as the Global Positioning System (GPS) [2-3]. Additionally, with the proliferation of Internet of Things (IoT) technology, analysts and programmers are using smartphones to perform transparent, automatic, and digital geographic isolation for isolated people over time. A typical technique is to 
approximate using a global tracking system and then check if the infected person is within the limited allowable area. While this approach is accurate, it requires great effort to pre-calibrate inside and outside the quarantine zone. This is expensive and inflexible for more spread quarantine sites [4]. GPS technology can enable three-dimensional location information. Information on latitude, longitude and altitude can be provided using four GPS satellite signals. Global Mobile Communications System (GSM) modules can be used to find the exact location of coordinates from GPS satellites [5]. Often, a tracking device is operated to detect the movement of people or objects. Relevant position information must be combined in a timely manner so that the movement can be displayed on the tracking system. There are multiple tracking systems, some based on the latency after the target is reached, while others are near real-time and real-time systems [6]. The Real-Time Online (RTO) system collects the real-time position of the satellite and continuously returns the data to the server. The general online RTO system depends on GPS / GPRS / 2G for data relayed to the server [7]. In [8], a tracking system for the COVID-19 infected peoples that reside in quarantine areas by using geo-fencing technology, is designed and implemented. The restriction of the areas and the prevention of unknown people from entering these areas are done by the monitoring stations. The approach used to detect vehicle deviations beyond the prescribed safety zone in [9] is based on GPS. In short, vehicles are marked using a digital stamp by applying a tracking system based on geo-fence technique. In [10], a transition from a mobile-based to an infrastructure-based geofencing system is made by utilizing mobile clients that adjust their location update strategies according to the recommendations of the infrastructure and the different activities of the mobile client user. The system implemented in [11] is a new application framework introducing the geonotification based services with the sentiment analysis. Combining GPS and GPRS in [12], the real-time tracking system analyzes the data received from the GPS receiver, providing the ability to track the current location or location of the vehicle at any specific time and date. In addition, information on vehicle status such as speed, mileage and driver performance is also provided. The vehicle tracking system of the research in [13] uses the Google maps service to track the vehicle, which has an account with posts of Google maps displaying vehicle location in real-time mode. A hardware module is built into the vehicle that uses GPS to detect the location and GSM to update the location in the vehicle account in the social network. The system in [14] is designed to prevent theft and make vehicle more secure by the use of GPS, GSM technology and a web application. Geofencing on the real-time GPS tracking system realized in [15] consists of two parts: the first part is used to receive GPS location data using the Sim908 device and send the data to a server using GSM and GPRS technologies, while the second part serves to display GPS data via web or Android application.

In this paper, a geofenced GPS system, adopting an efficient border protection system, is proposed for quarantine and monitoring purposes. It can be useful in monitoring infected individuals in quarantine, tracking vehicles, ships, and planes within geographical boundaries, etc. The microcontroller-based system includes GPS and GSM modules and is designed as a bracelet. The performance of the system is successfully tested under various location conditions.

\section{Theoretical Background}

\subsection{Global Position System (GPS)}

GPS is a satellite based navigation system. The GPS contains three significant segments. The space segment is the shape of satellites that rotate around the Earth in multiple space orbits, piling up to six or more space orbits. The control segment is the ground equipment that performs the task of controlling the space segment, telemetry, satellite tracking and maintaining the satellite orbital configuration. The user segment is created from satellite receivers and is used to receive signals and identify sites based on received signals [16]. The GPS satellite sends a one-way signal to the GPS receiver on the ground. The satellite determines the location of the receiver and the time at which the signal is sent, as the timing information plays a major role in determining the location of the user. Therefore, the GPS contains an atomic clock to provide a time reference accurately [17]. The distance $d$ between a particular GPS satellite and the receiver can be found from the travel time $t_{t}$ of a signal from the satellite to the receiver as follows,

$$
d=t_{t} \mathrm{c}=\left(\mathrm{t}_{s r}-t_{s t}\right) c
$$

where $t_{s r}, t_{s t}$ and $c$ are signal reception time, signal transmission time, and speed of light, respectively.

The actual position of the GPS receiver is obtained based on the GPS signal transit time from three nearby satellites and their precise trilateration (triangulation) of space coordinates. However, four satellites are required rather than three, in order to determine one's location in 3D space [18].

\subsection{Geofencing}

The geofencing technique consists of a mixture of two operations, which are the frequent tracking of the location of a mobile device and the description of a geographical of interest region [2]. Geofencing collects information about the user's actual location, taking into account whether the user is close to places that might be relevant. To mark an actual location, it must assign its latitude and longitude. Latitude, longitude, and radius are determined by a geofence that creates a ring area or 
fence around the entire monitoring site. This includes constantly checking and aligning mobile device location with a set of practical boundaries, i.e. geofences [19-20].

\section{System Overview}

In this section, the devices, libraries and algorithms used in the establishment of the system are introduced in general along with their working principles.

\subsection{System Implementation}

The developed tracking system consists of a GPS L86 module, SIM800c GSM module, and microcontroller within the Arduino UNO board, which is utilized as the processing unit for the tracking system. The GPS receiver determines the location of the person i.e. the patient. Afterward, the obtained location data is sent to the server (adfruit.io) via GSM. Fig. 1 shows the circuit diagram of the tracking system.

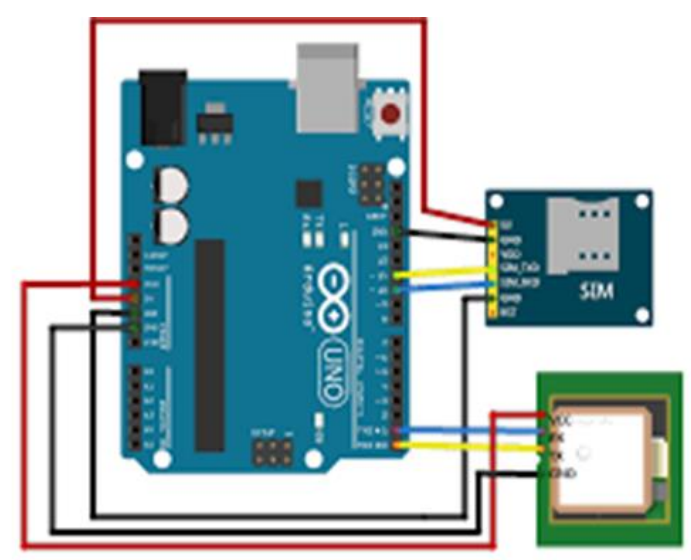

Figure 1: Interfacing GSM and GPS Module Using Arduino

COVID-19 infected individuals are tracked with a special application implemented using the MIT App Inventor platform. The application is compatible with smartphones running Android operating system. People who carry the recommended tracking system (in the form of a bracelet) can be tracked using this application. Therefore, a geofence technology is implemented to only allow the infected person to move within the geofence. Moreover, with the developed application, the dimensions of the geofence can be completely controlled, in other words, it can be enlarged or reduced.

\subsection{System's Working Principle}

To obtain the latitude and longitude of a particular location, a practical test is first performed using the GPS module which is connected to the ATmega328P SMD microcontroller chip surface mounted in Arduino UNO. Two libraries named "SoftwareSerial.h" and "TinyGPS.h" are embedded with Arduino IDE for programming the code. The flow chart of the
GPS working principle is shown in Fig. 2. The GPS receiver collects the longitude and latitude data, then the Arduino IDE serial monitor displays the received location data.

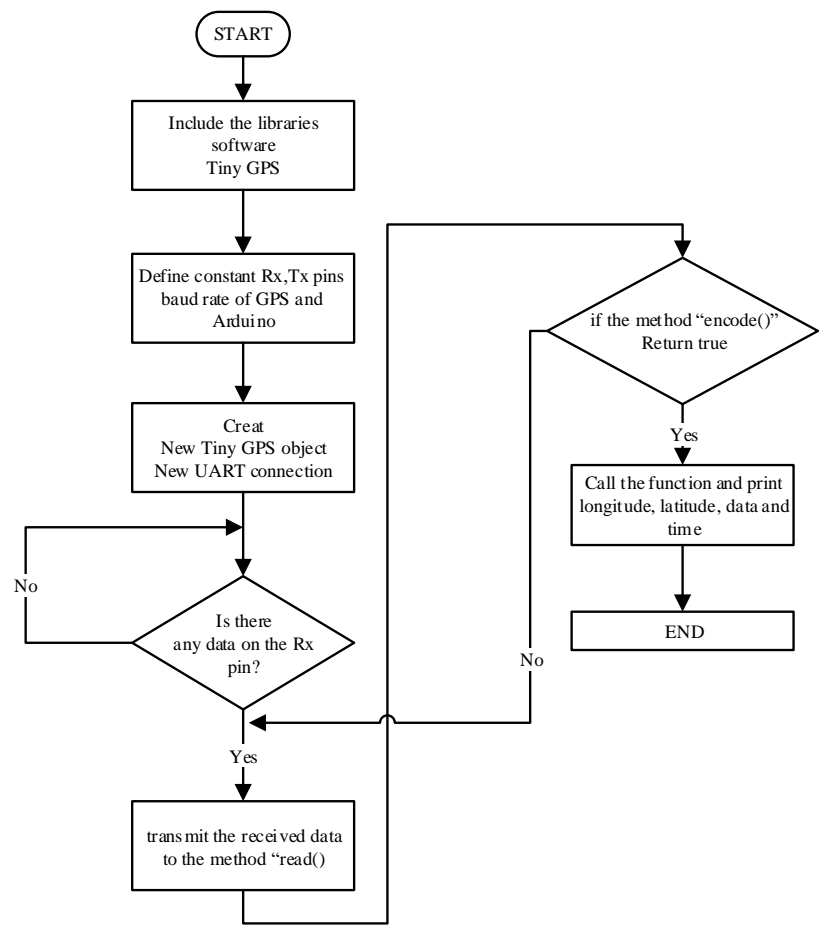

Figure 2: Working principle of the GPS module

In the GSM network, a SIM800 GSM module and the ATmega328P microcontroller chip are used to transmit data to the Adafruit server. In practice, the person holding the proposed tracking system (i.e. bracelet) does this. AT command mode is used for programming data transfer commands. The flow chart for the GSM operation is shown in Fig. 3. 


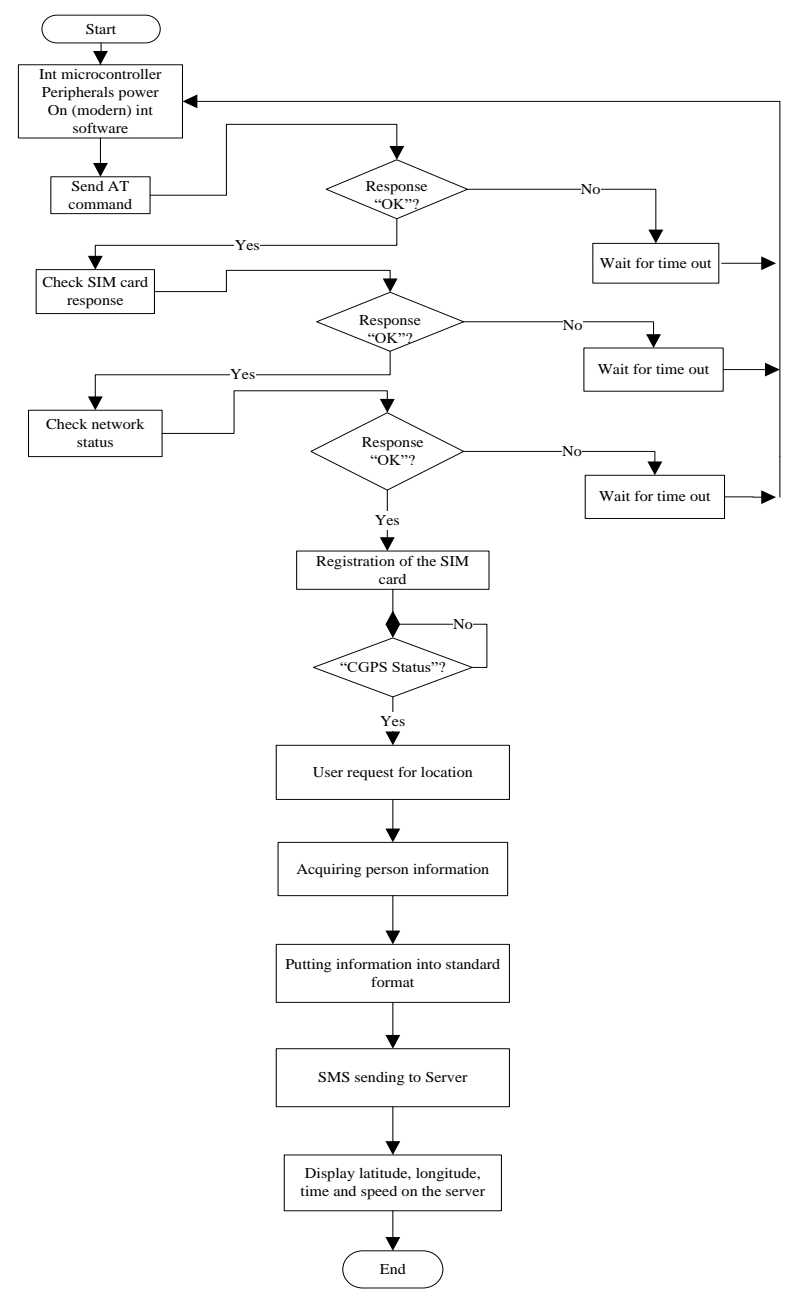

Figure 3: Working principle of GSM module

AT commands can be used to test the connection between the operation of the GSM module and the cell phone base station. For geolocation data (latitude, longitude, time and speed), the receiver receives the information of the GPS signal and then sends this information to the server. Fig. 4 shows the flowchart of sending and receiving data.

\subsection{The Server (Adafruit.Io)}

Adafruit.io cloud service is used to view the data in real time. The server port number 1883 is used when setting the hostname to asio.adafruit.com in the Arduino IDE. The code consists of Adafruit account username, regenerable special AIO key, feed setup and feed name, SSID, and internet port password. The feed name is the subject name that can be subscribed or published for internet connection through the server. To properly establish the relationship connecting the Arduino and the server, the code is compiled and sent to the control. It also shows the definition of the server name, server port, and key assigned to the server.

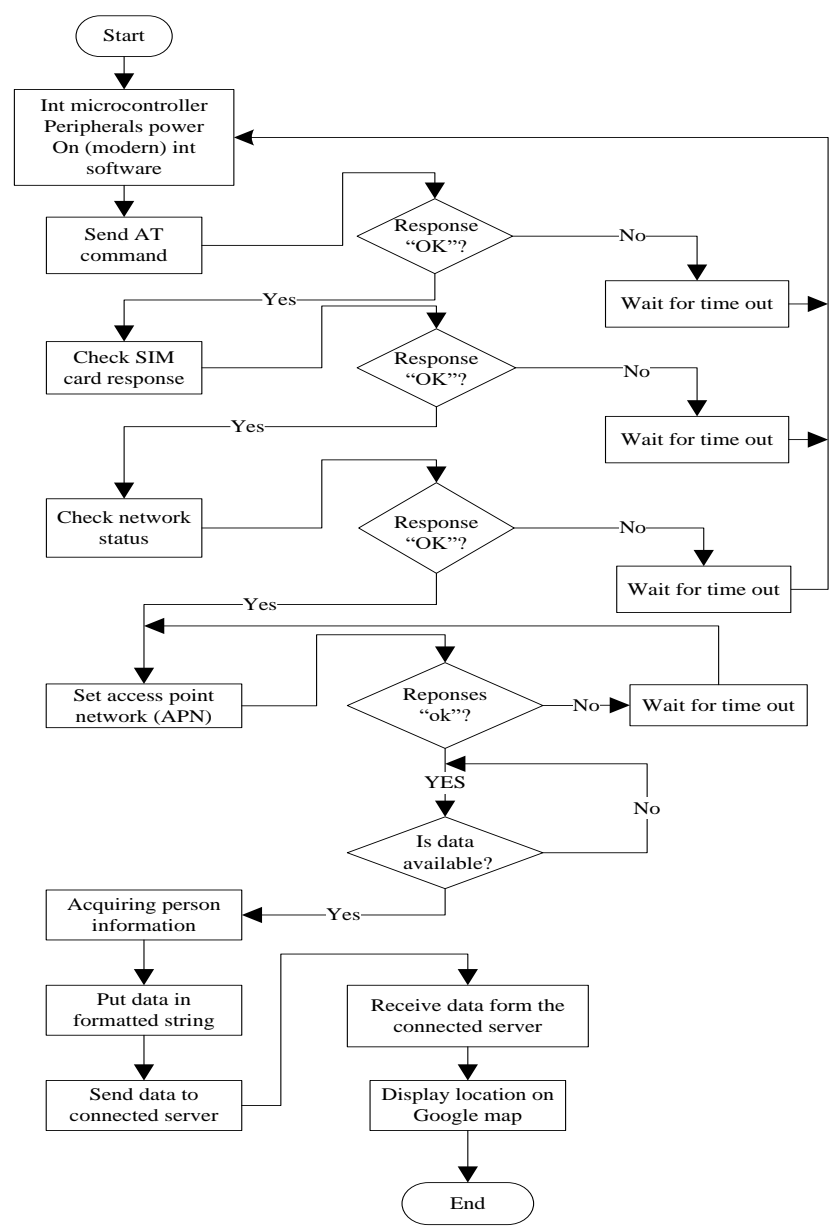

Figure 4: Flowchart of sending and receiving data

\subsection{Geofencing Application}

In this study, geofencing incorporates the actual position of the patient with the proximity of the patient to the quarantine site. The quarantine site is marked by specifying its latitude and longitude while the proximity to the defined site is determined by adding a radius. Thus, the applied geofencing is in a circular shape [9]. The geo-fencing here is applied via the MIT App Invertor for Android. The proposed system is designed and implemented to meet the need for a proper quarantine as well as the ability to locate, and adjust quarantine site boundaries with this Android app. It also gives a warning on the exit or entrance of the patient carrying the tracking device (in this case a bracelet), as shown in Fig. 5. 


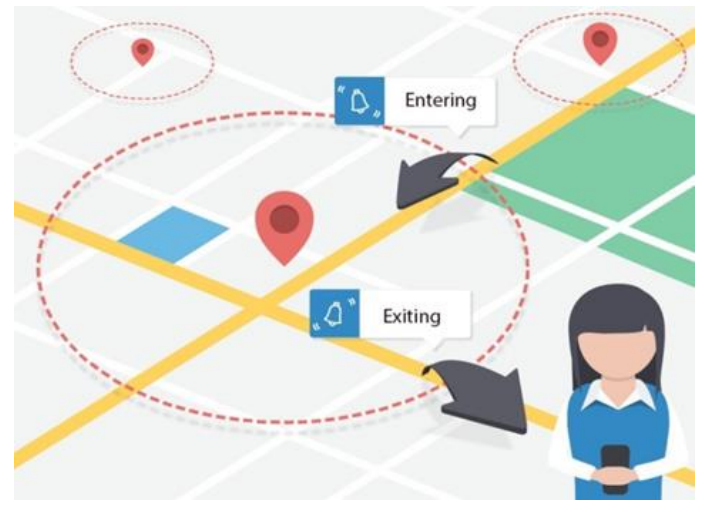

Figure 5: Applied circular geofencing

To summarize, with the geofencing based tracking system, first the location data (longitude and latitude) is received from the satellites by the GPS receiver and then transferred to the ATmega328P microcontroller for further processing, and then the obtained data is transmitted by the GSM module to the Adafruit server to be displayed in the mobile application.

\section{System Implementation and Results}

\subsection{Tracking Device - Bracelet}

Fig. 6 shows the block diagram of the bracelet design used as the tracking device.

The circuit of the designed tracking system - the bracelet includes the microcontroller, GPS and GSM modules given in Fig. 1 altogether. The bracelet circuit contains GPS\&GSM (SIM800C) modules, ATMEGA328P microcontroller, Common Mode Filter (ZJYS51R5), Switcher (LM2596), voltage regulator (SPX3819), a set of Headers, GPS and GSM antenna (IPEX3), a set of LEDs, and an IC Charge (MCP73831) unit. The double-sided Printed Circuit Board (PCB) of the bracelet is shown in Fig. 7.

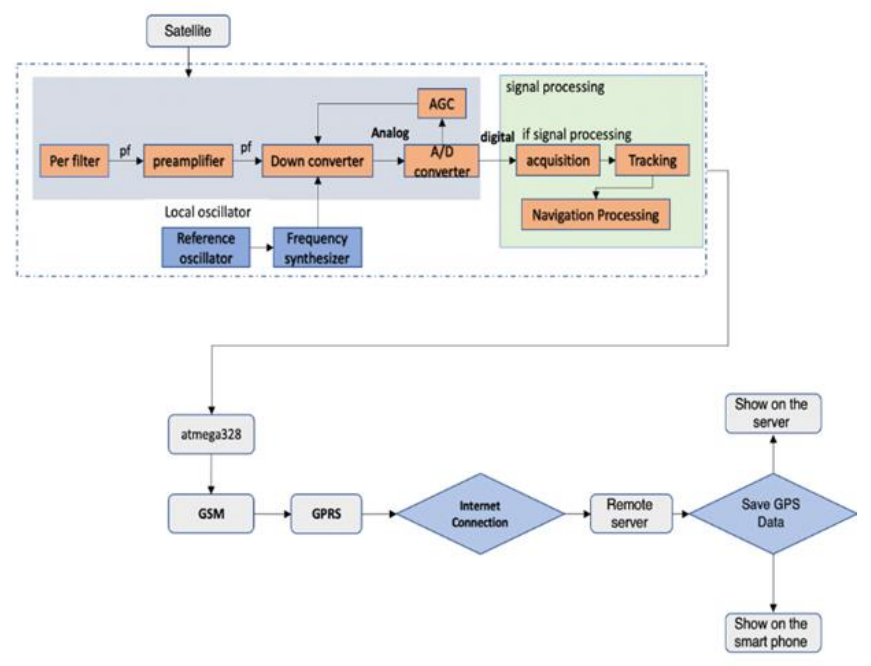

Figure 6: Block diagram of the tracking device - bracelet

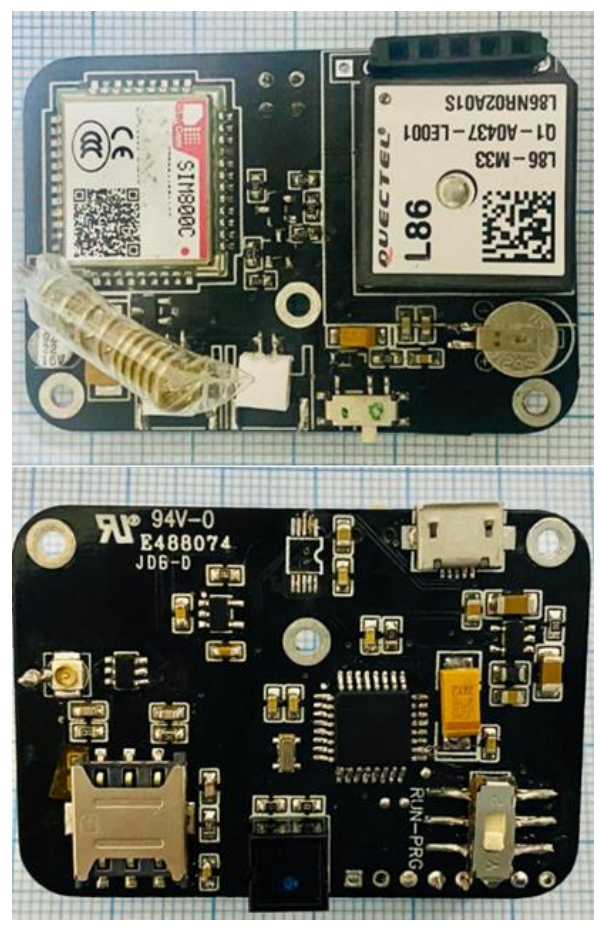

Figure 7: PCB of the implemented tracking device - bracelet

\subsection{Web Server and Database}

The microcontroller is connected to the SIM800 module of the GSM shield, to act as an SMS transceiver. In general, the tracking device sends an SMS condition request constantly to the unit, at any time the central server wants to find the patient. The GPS inside the bracelet sends the received data continuously to the server via GSM. This data includes the coordinates (latitude and longitude), time, and speed. In a smartphone application, the server derives the coordinates from the data, which is received by SMS. The patient's location is displayed on the mobile phone map and archived in the database for potential future use. The web server is linked to the database, which contains information about the patient's location. A sample of experimental data of patient location information obtained from the database based on a test run is listed in Table 1. 
Table 1. Real-time patient location information.

\begin{tabular}{|c|c|c|c|c|}
\hline TIME & DATA & LAT & LON & ALT \\
\hline $16: 27: 22$ & $30-12-2020$ & 40.986855 & 28.708899 & 98 \\
\hline $16: 27: 24$ & $30-12-2020$ & 40.986865 & 28.708908 & 98 \\
\hline $16: 27: 26$ & $30-12-2020$ & 40.986878 & 28.708914 & 98 \\
\hline $16: 27: 28$ & $30-12-2020$ & 40.986886 & 28.708921 & 98 \\
\hline $16: 27: 30$ & $30-12-2020$ & 40.986894 & 28.708928 & 98 \\
\hline $16: 27: 32$ & $30-12-2020$ & 40.986907 & 28.708933 & 98 \\
\hline $16: 27: 34$ & $30-12-2020$ & 40.986915 & 28.708941 & 98 \\
\hline
\end{tabular}

Experimental results show that the minimum time for updating location information is $2-4$ seconds. This time can be configured according to the individual movement.

\subsection{Geofencing Performance Validation}

The application is run on Android phones and two types of commands are executed, 'fence' and 'patient'. The 'fence' command specifies the geo-fence, while the 'patient' command specifies the bracelet holder location. Apart from the geo-fence and the patient's location, the distance and speed of the patient to the geo-fence are also displayed on the screen. The tracking system automatically updates the patient's location every 3-5 seconds. To validate the performance of the geofencing based tracking system three different scenarios are examined.

In the first scenario shown in Fig. 8, the blue marker and red marker/circle represent the patient wearing the bracelet and the geographic boundary for quarantine, respectively. It can be observed that a green message indicates that the patient is within the geofencing boundaries, that is, within the quarantine area. In the second scenario shown in Fig. 9, when the patient crosses the geofence, the distance between the patient's location and the geofence is calculated. An alert is sent to the mobile phone and a red message indicates that the patient is outside the quarantine area. The alert works continuously until the distance between the patient and the geofence equals zero, meaning that the patient is within the quarantine zone when it stops.

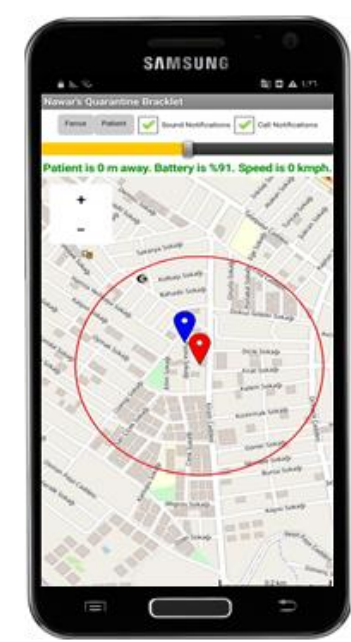

Figure 8: Patient located within the geofence

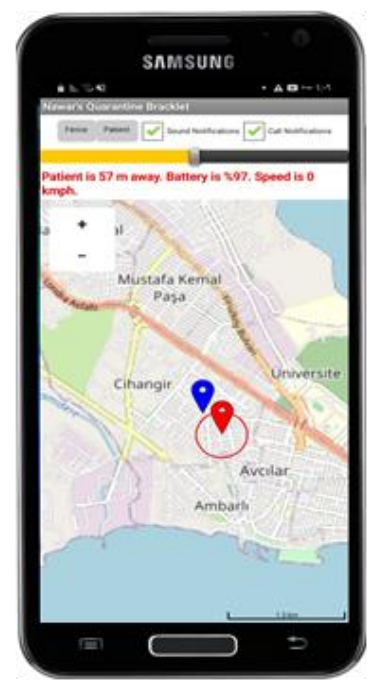

Figure 9: Patient located outside the geofence

The third scenario illustrates the ability to change the boundaries of the geofence. Thus, the diameter of the geofence can be adjusted according to the specified area for monitoring. The tracking device is programmed with maximum and minimum distances of $1 \mathrm{~km}$ and $200 \mathrm{~m}$, respectively. Fig. 10 shows the patient's position within the increased boundaries of the geofence. 


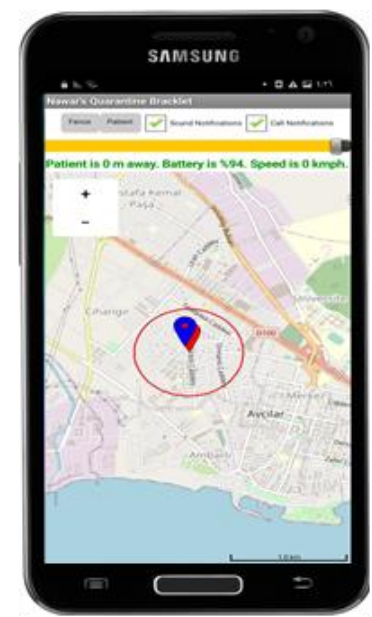

Figure 10: Enlargement of geographic boundary

The bracelet has a solid design, so it can be used in different climatic conditions and is connected to the mobile phone using the cloud. The complete tracking system (bracelet and mobile device) is shown in Fig. 11.

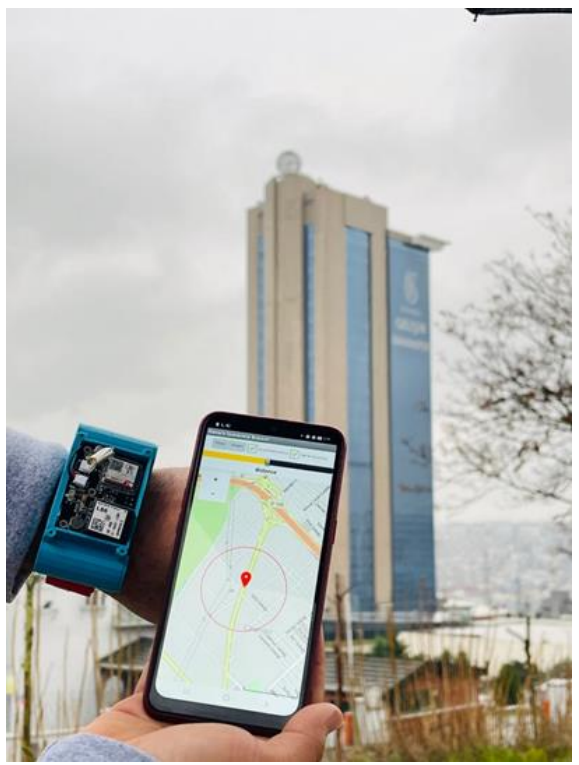

Figure 11: Complete system (bracelet and mobile device)

\section{Conclusion}

A real-time tracking system based on geofence technology has been demonstrated and developed. The tracking system has been proposed as an alternative to the recently required strict quarantine policy. It consists of a bracelet containing GPS\&GSM modules and a microcontroller, and a mobile device. People infected with COVID-19 are tracked with a special application implemented using the MIT App Inventor platform. The application is compatible with smartphones running the Android operating system. For geolocation data (latitude, longitude, time and speed), the receiver receives the
GPS signal information and then sends this information to the web server.

Geofencing technology provides accurate real-time information that enables the person responsible for monitoring to quickly track the person leaving the specified area. An alert is sent to the mobile phone when the patient crosses the boundaries of the geofence. The performance of the implemented tracking system has been verified by experiments under various conditions.

\section{References}

[1] A. A. M da Silva, 'On the possibility of interrupting the coronavirus (COVID-19) epidemic based on the best available scientific evidence', Rev Bras Epidemiol, 23, e200021, 2020.

[2] F. Reclus, and K. Drouard, 'Geofencing for fleet \& freight management', 9th International Conference on Intelligent Transport Systems Telecommunications (ITST), Lille, France, pp. 353-356, October 20-22, 2009.

[3] R. R. Oliveira, et al. 'SWTRACK: An intelligent model for cargo tracking based on off-the-shelf mobile devices' Expert Systems with Applications 40, pp. 2023-2031, 2013.

[4] J. Tan, et al. 'IoT Geofencing for COVID-19 Home Quarantine Enforcement', IEEE Internet of Things Magazine 3.3, pp. 24-29, 2020.

[5] U. Bharavi, and R. M. Sukesh. 'Design and development of GSM and GPS tracking module', 2nd IEEE International Conference on Recent Trends in Electronics, Information \& Communication Technology (RTEICT), India, pp. 283288, May 19-20, 2017.

[6] E. Z. Adwan, 'Real Time Cost Effective Of People Tracking System', Master Thesis, Islamic University Gaza, Palestine, 2015.

[7] S. Namkhun, and D. Hormdee, 'Two-Way Semi-Offline Location Tracking and Control System via GSM', ITCCSCC, Gyeongju, Korea, pp. 476-479, June 19-22, 2011.

[8] R. Anto Arockia Rosaline, et al. 'Tracking the Covid zones through geo-fencing technique', International Journal of Pervasive Computing and Communications, Emerald Pub. Ltd., 2020.

[9] D. Suganthi, et al. 'Vehicle tracking with geofencing on Android platform', International Journal of Engineering Science and Technology, pp. 16992-16995, 2018. 
[10] S. W. Rahate, and M. Z. Shaikh, 'Geo-fencing infrastructure: location based service', International Research Journal of Engineering and Technology, Vol. 3, No. 11, pp. 1095-1098, 2016.

[11] P. Mohite, et al. 'Geofencing and location based reminder services', International Journal of Advance Engineering and Research Development, Vol. 3, No.10, pp. 137-141, 2016.

[12] W. El-Medany, et al. 'A cost effective real-time tracking system prototype using integrated GPS/GPRS module', 6th International Conference on Wireless and Mobile Communications (ICWMC), Valencia, Spain, pp. 521-525, September 20-25, 2010.

[13] A. ElShafee, et al. 'Integrating social network services with vehicle tracking technologies', International Journal of Advanced Computer Science and Applications Vol. 4, No. 6, pp. 124-132, 2013.

[14] P. Verma, and J. S. Bhatia, 'Design and development of GPS-GSM based tracking system with Google map based monitoring', International Journal of Computer Science, Engineering and Applications (IJCSEA), Vol. 3, No. 3, pp. 33-40, 2013.

[15] Z. Ozdemir, and B. Tugrul, 'Geofencing on the Real-Time GPS Tracking System and Improving GPS Accuracy with Moving Average, Kalman Filter and Logistic Regression
Analysis', 3rd International Symposium on Multidisciplinary Studies and Innovative Technologies (ISMSIT), Ankara, Turkey, pp. 1-6, October 11-13, 2019.

[16] R. J. Bates, 'GPRS: general packet radio service', McGraw-Hill Professional, 2001.

[17] R. Bajaj, et al. 'GPS: location-tracking technology', Computer, 35(4), pp. 92-93, 2002.

[18] B. Lee, et al. 'Development of self-localizer using collaboration of trilateration and triangulation', 11th International Conference on Fuzzy Systems and Knowledge Discovery (FSKD), Xiamen, China, pp. 729733, August 19-21, 2014.

[19] S. R. Garzon, et al. 'Infrastructure-assisted geofencing: proactive location-based services with thin mobile clients and smart servers', 3rd IEEE International Conference on Mobile Cloud Computing, Services, and Engineering, San Francisco, CA, USA, pp. 61-70, March 30-April 3, 2015.

[20] S. R. Garzon, and B. Deva, 'Geofencing 2.0: taking location-based notifications to the next level', ACM International Joint Conference on Pervasive and Ubiquitous Computing, Seattle, WA, USA, pp. 919-930, September 13-17, 2014. 